\title{
Se préparer au métier de footballeur : analyse d'une socialisation professionnelle
}

\section{STAPS, 2008, n ${ }^{\circ} 82$, pp. 29-42}

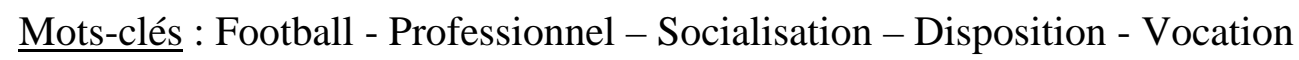

\section{Résumé :}

Cet article, issu d'une thèse de sociologie, explore le mode de formation des footballeurs professionnels. L'étude porte sur la structure de formation d'un grand club de la première division du championnat de France qui accueille de jeunes footballeurs âgés de 12 à 19 ans. La socialisation et la production des dispositions sur lesquelles s'appuie cette entreprise de formation sont appréhendées grâce à une enquête socio-ethnographique qui mêle entretiens avec les apprentis et les formateurs (58), observations de longue durée et analyse de dossiers scolaires. Sur la base de ce matériau peuvent être mis en lumière les traits caractéristiques d'une expérience de formation fondée sur un engagement à la fois intense et incertain. Deux axes d'analyse sont alors développés. D'une part, le texte expose les conditions et les effets d'un investissement intensif vécu sur le registre de la "passion ». L'apprentissage suppose l'intégration à un «monde à part » qui implique une recomposition des appartenances nonsportives (scolaires, familiales, amicales). D'autre part, cette absorption par l'univers footballistique entraîne une modification du rapport à la pratique qui, du fait d'une familiarisation aux enjeux concurrentiels et professionnalisant, tend progressivement à être pensé en termes de carrière individuelle.

Title : Preparing to be a footballer : analysis of a professional socialization

$\underline{\text { Key-words }}$ : Football - Professional - Socialization - Disposition - Vocation

Abstract : This article, stemming from a thesis of sociology, explores the training process of professional footballers. The study deals with the training centre of a professional club, playing in the first league of the French championship, which welcomes young footballers aged 12 to 19 . The socialization and the production of dispositions on which the training procedure is based, are comprehended thanks to a socio-ethnographic enquiry that mixes interviews with the apprentices and the trainers (58), long-term observations and the analysis of school records. This material permits to enlighten the characteristic features of their experience, founded on an intensive but also uncertain investment. Two main lines of analysis are developed. On the one hand, the article exposes both the conditions and the effects of an intensive investment perceived as a "passion". The training supposes the integration to a "world apart" that implies the recomposition of the non-sportive belonging (school, family, friends). On the other hand, this absorption by the footballistic universe leads to a modification of the connection to practice : indeed, due to the familiarization with competitive and professionalizing stakes, the latter tends to be progressively thought in terms of individual career. 


\section{Se préparer au métier de footballeur : analyse d'une socialisation professionnelle}

L'observation sur la longue durée révèle les mutations qu'a connu le marché du travail footballistique. Mobilité professionnelle et individualisation du rapport des joueurs à leur club ont consacré un nouveau rapport au métier (Slimani, 2000). Les travaux socio-historiques montrent de quelle manière les footballeurs sont passés, au tournant des années 1980, d'une situation de forte dépendance à l'égard des clubs, cristallisée dans des formes de contrats très contraignantes (dont les «contrats à vie » ont été l'incarnation la plus exemplaire (Wahl \& Lanfranchi, 1995)) à une emprise de la logique du marché (Faure \& Suaud, 1999). Une des conséquences directes de l'individualisation du marché du travail est le déplacement des enjeux des luttes collectives (via le syndicat des joueurs, l'Union Nationale des Footballeurs Professionnels (UNFP)) vers les négociations individuelles entre joueurs et clubs (par l'intermédiaire des agents sportifs) : «D’une formule, on pourrait opposer les générations 60 70 à celles des années 80-90 en disant que le marché atomise ce que les affrontements individuels et collectifs - avaient contribué à unir » (Faure \& Suaud, 1999, 235). L'objet de ce texte, issu d'une thèse de sociologie (Bertrand, 2008), est alors d'analyser de quelle manière sont «fabriqués » ces nouveaux professionnels français dont le succès dans les grandes ligues européennes demeure conséquent (Poli, 2007).

$\mathrm{Au}$ cœur de la démarche entreprise se trouve l'articulation entre un point de vue et une échelle d'observation qui singularisent les analyses menées. La construction de l'objet se fonde, en effet, sur l'adoption d'un angle d'approche, celui de la socialisation, et le choix d'une approche locale des phénomènes. Le choix de la délimitation de l'enquête au sein d'un seul club permet une investigation intensive et socio-ethnographique de l'expérience de formation. Ce que l'approche perd en profondeur temporelle par rapports aux travaux sociohistoriques précédents, notamment dans l'étude de la sociogenèse d'un espace social spécifique du football professionnel (Faure \& Suaud, 1999; Slimani, 2000; Wahl \& Lanfranchi, 1995), elle le gagne en richesse des analyses des mécanismes de socialisation. Alors que ces travaux ont fait porter leurs plus gros efforts empiriques sur les questions de structuration de ces univers parce qu'ils s'interrogent prioritairement sur l'évolution de l'organisation de ces pratiques (en analysant les rapports de force entre les différents acteurs qui participent à la régulation de ce secteur (acteurs publics, dirigeants fédéraux et des clubs, joueurs professionnels)), le changement d'échelle permet un accroissement cumulatif des 
connaissances. Dans cette optique, le travail vise à analyser les modalités de construction de dispositions à l'intérieur d'une structure de formation, celle d'un grand club de la première division du championnat de France. A partir de l'analyse des récurrences des pratiques et des discours sont reconstitués ces principes générateurs que sont les dispositions, «manières plus ou moins durables de voir, de sentir et d'agir » (Lahire, 2002, 19), dont l'incorporation repose sur des expériences répétées. C'est à travers ce concept, qui permet d'opérationnaliser l'étude de la socialisation, qu'est menée l'analyse de l'appropriation de la condition d'apprenti footballeur et de la manière dont se construit un rapport au métier adéquat à ce marché du travail, qui suppose tout à la fois un engagement vocationnel et l'intériorisation de la régulation concurrentielle et individualisante.

Le travail s'appuie sur une enquête ethnographique menée entre 2001 et 2004 (cf. encadré : «L'enquête et sa méthodologie ») à l'intérieur de ce club qui sélectionne et forme environ cent trente jeunes footballeurs engagés en «préformation » (entre 12 et 14 ans) puis en formation (15-19 ans). Ces derniers connaissent dans la structure un apprentissage sportif intensif (de 4 à 7 entraînements hebdomadaires) associé à une scolarité aménagée. Trois principaux matériaux sont à l'origine des analyses : des entretiens (58) dont trente-trois ont été réalisés avec des aspirants professionnels âgés de 16 à 19 ans; environ 200 séances d'observations menées à l'intérieur du club (soit entre 500 et 600 heures de présence dans la structure) ; et enfin, l'analyse de trente-six dossiers scolaires de jeunes membres de cette école du football. Durée de l'enquête, pluralité des points de vue récoltés et variation des lieux d'observation ont permis de saisir les situations quotidiennement vécues par les apprentis sportifs tout en limitant les effets de perturbation liée à l'intrusion extérieure que constituait notre présence. C'est sur la base de ces données que l'étude montre les transformations du rapport des jeunes joueurs à leur pratique au cours de la formation. Pour rendre compte de cette préparation au métier de footballeur, il est d'abord nécessaire de souligner la capacité de cette formation à aspirer les apprentis footballeurs dans un engagement intensif. Une première partie permet ainsi de décrire cette absorption par le projet de professionnalisation que permet l'entrée dans une institution aussi enveloppante que le centre de formation. Outre son intensité, l'engagement dans cette course à des enjeux spécifiques se caractérise par la double temporalité dans laquelle sont immergés les enquêtés. Dans un deuxième temps, l'analyse de cette dualité entre un temps compétitif et des échéances de professionnalisation individuelles permet de souligner la manière dont les apprentis sont amenés à s'approprier les conditions d'un marché du travail atomisé. 


\section{L'enquête et sa méthodologie}

Afin de travailler de manière systématique la socialisation professionnelle, le parti pris méthodologique a consisté en l'exploitation intensive d'un seule structure de formation. Elle comporte trois volets principaux.

Entretiens : L'objectif des entretiens avec les apprentis était de solliciter des récits de pratiques détaillés (la durée moyenne des entretiens est proche de deux heures), des souvenirs de situations qui obligent le moins possible à rompre avec l'expérience, avec l'ambition d'éviter les discours plus généraux de présentation de soi davantage susceptibles d'être investis par un discours de la vocation. Les thèmes abordés étaient les suivants : les parcours sportifs antérieurs (initiation, clubs, sélections, etc.), le parcours scolaire, l'apprentissage dans le Centre, le capital corporel et sa gestion, la professionnalisation, les usages du temps libre et les loisirs, les relations de sociabilité, les propriétés sociales et sportives de leur famille. En complément, des entretiens ont été réalisés avec des parents d'apprentis enquêtés (quatre) et d'anciens pensionnaires du Centre (deux cas). De plus, tous les intervenants (sportifs, médicaux, scolaires) ont été rencontrés $(n=16)$, l'enjeu principal était alors de repérer les catégories de perception et de jugements qui structurent la manière dont ils produisent la formation et sélectionnent les aspirants footballeurs en sollicitant, principalement, des récits sur les joueurs en apprentissage et les savoir-faire transmis. Entraînement (programme, composition des séances, qualités et savoir-faire exigés, règles de discipline, etc.), compétitions (composition de l'équipe, choix des postes, titulaires et capitaines, notation des joueurs, etc.), suivi scolaire et parcours personnel (professionnel, scolaire et sportif) constituaient les thématiques des rencontres avec les entraîneurs.

Observations : L'enquête a consisté dans le suivi en trois étapes de plusieurs niveaux de formation: d'un groupe de joueurs âgés de seize et dix-sept ans (deux mois et demi), de l'équipe des « 12 ans » (environ quatre mois et demi), puis des équipes en fin de formation et plus particulièrement de celle des « 18 ans » (un an). Si les terrains de jeux sont restés les lieux les plus accessibles (entraînements et matchs), d'autres espaces ont pu être progressivement et partiellement investis (vestiaires, salle de soin, déplacement en matchs, lycée). Les thèmes privilégiés d'observation étaient : l'organisation pédagogique (matérielle, type d'exercices et de savoir-faire), les jugements et corrections des entraîneurs, les catégories d'évaluation des gestes, le mode d'exercice de l'autorité, la place de la compétition, la gestion et les représentations du corps, les interactions entre joueurs.

Analyse des dossiers scolaires : Si les entretiens réalisés avec les apprentis footballeurs et les observations (en tant que surveillant d'externat) constituaient une première source pour analyser l'évolution des pratiques et rapports à l'école, l'analyse des dossiers scolaires, malgré leur inégal contenu, a constitué un outil efficace d'objectivation. La reconstruction des parcours s'est appuyée sur le recueil des données disponibles dans les bulletins : l'itinéraire scolaire (orientation, redoublements, diplômes), les notes moyennes annuelles (moyenne générale et d'EPS) et leur écart à la moyenne de la classe, les sanctions positives et négatives décernées par les conseils de classe («Félicitations», «Avertissements», etc.), les «observations» récurrentes des enseignants et les absences. 


\section{La production d'un engagement intensif}

La formation de footballeurs de haut niveau prépare et s'appuie sur un engagement intensif vécu comme une «passion » et souvent pensé, aussi bien chez les apprentis que pour les formateurs, à travers le langage de la vocation. L'étude de cet apprentissage permet de montrer la capacité de l'institution à susciter cet investissement, les mécanismes et les effets de cette captation malgré l'incertitude des débouchées offerts (1).

\subsection{Vivre dans une « bulle »}

\subsubsection{L'intégration à un « monde à part »}

Revenant sur son parcours dans le centre de formation, un ancien pensionnaire reconverti depuis plusieurs années dans un autre domaine professionnel décrit son expérience à partir d'une opposition très significative entre la vie « réelle », «normale », le « vrai » monde, et l'existence «irréelle », «déconnectée » de l'apprenti footballeur. La vie d'aspirant se distingue selon lui par son caractère exceptionnel et par l'emprise de cet espace et de ses enjeux, lui faisant dire qu'il vivait dans une «bulle », dans un «microcosme »: «le football, c'est ce qui te fait aller, c'est ce qui te fait vivre, ce qui te fait être bien, pas bien. (...). On est hors du temps quoi. Y a une autre dimension qui se crée ». Sa sortie de l'univers footballistique lui permet une distance face à cet engagement («on se rend pas compte », ditil souvent pour évoquer cette époque) et le porte à considérer cette vie comme presque étrangère à lui-même. Cette impression de mener une vie à part, à l'intérieur d'un monde clos, souvent soulignée chez les sportifs de haut niveau (Fleuriel, 2004, 57 ; Papin, 2007 ; Brissonneau, Aubel \& Ohl, 2008) est révélatrice du degré d'emprise de cette «bulle» qu'entraîne la socialisation footballistique. Celle-ci repose, en effet, sur l'immersion dans un temps et un espace proprement sportifs.

Le parcours de formation des apprentis ressemble ainsi à un processus d'absorption progressive par des enjeux qui transforment le temps sportif en temps structurant et assure l'adhésion au projet professionnel. L'entrée dans la formation correspond, en effet, à l'insertion dans un monde à part dans lequel les joueurs sont amenés à vivre dans un univers séparé, un «contre-espace » et un «contre-temps » (Faure \& Suaud, 1999, 199). L'inflation du temps d'entraînement et l'accentuation des contraintes d'hygiène de vie, intériorisées sous la forme d'un souci de préservation du capital-corps, facilitent la subordination des autres temps sociaux à l'urgence sportive. Les jeunes apprentis intègrent comme lieu de vie (plus de la moitié sont internes), ou comme lieu de pratique, un espace construit autour de sa fonction 
sportive. Cet espace, qui réunit la formation et l'entraînement des professionnels, est aussi vécu comme un lieu «à part » faisant de son appropriation une dimension de l'appropriation symbolique du métier. Ainsi, fréquenter le centre de formation, c'est d'abord entrer en contact avec les professionnels du club et bénéficier d'installations très supérieures à celles des clubs précédemment traversés. A l'inverse des quartiers stigmatisés symboliquement, qui dévaluent leurs habitants et que ceux-ci ont tendance à dégrader en retour (Lepoutre, 1997, 51), l'espace « riche» du Centre invite les joueurs à en être «dignes », à se montrer à la hauteur d'une appartenance si distinctive. Cet espace se caractérise aussi par son degré de fermeture, une clôture qui croît à mesure que les joueurs progressent dans la hiérarchie du club (les incursions extérieures sont fortement limitées ou encadrées par un service d'ordre lors des entraînements de l'équipe première). Cette fermeture matérialise, en creux, le fait qu'entrer dans le club est un droit réservé à une minorité. S'actualise ainsi dans la configuration spatiale un rapport avec l'extérieur et les profanes qui participe, en même temps qu'il en est le produit, à l'intériorisation chez les membres du club du sentiment d'occuper une position extraordinaire.

\subsubsection{Les conditions sportives d'entrée en formation}

Entrer dans une «bulle» apparaît ainsi comme la condition et la manifestation d'un engagement intensif dans l'activité sportive et cette entrée suppose l'appropriation d'un espace et d'un temps propre. Il faut cependant souligner que cette intégration des jeunes apprentis dans l'univers footballistique n'a des chances de succès que parce qu'elle s'adresse à une population déjà fortement sélectionnée. En effet, ce n'est qu'à l'issue d'une longue pratique amateur durant laquelle, à force d'élections et de gratifications, les jeunes enquêtés ont acquis le sentiment d'être «doués » qu'ils franchissent ce cap décisif dans le recentrement sur le jeu footballistique. C'est ce que montre l'analyse, que permet la dimension rétrospective des entretiens menés, du temps où ce sport n'était encore qu'un jeu pour eux.

Familiarisés précocement à la pratique (plus de la moitié des joueurs enquêtés sont entrés dans un club un ou deux ans avant l'âge de six ans), les apprentis ont souvent été initiés à la pratique par un père lui-même pratiquant (deux tiers des pères ont joué en club et un tiers a pratiqué dans des championnats nationaux (entre les divisions professionnelles et le niveau de CFA2)). L'imprégnation par une culture footballistique au sein des familles investies dans cette pratique (notons, par exemple, que neuf frères sur dix des enquêtés ont eux-même pratiqué ce jeu) constitue un déterminant important de ces parcours. L'adhésion et le goût pour cette pratique s'est trouvé renforcé par une ascension sportive précoce (reconnaissances par les entraîneurs, sollicitations de clubs plus huppés, sélections fédérales). S'ils débutent 
pour la majorité d'entre eux à l'intérieur de clubs modestes dans lesquels prime l'interconnaissance locale, ils s'entraînent souvent dès l'âge de onze ans dans des clubs d'élites (les deux tiers évoluent dans des associations participant aux championnats régionaux ou nationaux jeunes). «C'était déjà plus sérieux» disent-ils souvent pour décrire sommairement cet accès à des niveaux de jeu plus élevés et la transformation de leur activité qui l'accompagne. Parce qu'ils sont reconnus sur ce marché amateur, l'orientation vers un club professionnel devient un avenir pensable et l'invitation de celui-ci a toutes les chances d'apparaître comme un appel à se réaliser soi-même. Ce n'est ainsi qu'à l'issue de ce parcours d'ascension et de sélection progressive que les enquêtés pénètrent l'univers footballistique professionnalisant. Mais si l'engagement de ces apprentis footballeurs doit être perçu au regard de leur parcours antérieur, mesurer cet investissement suppose également de diriger l'analyse vers ses effets sur les autres pans de leur existence.

\subsection{Les effets de l'emprise sportive : la recomposition des appartenances}

Rendre compte de cette absorption progressive par les enjeux sportifs suppose d'observer les effets de l'engagement sur les autres univers d'appartenance et relations des apprentis. En effet, si l'intensité de l'engagement exigé rend problématiques les investissements sociaux parallèles, elle conduit davantage à une emprise qu'à un enfermement strict, au sens d'une appartenance exclusive à l'univers footballistique. La «bulle » n'est donc pas totalement close et hermétique car cette assignation à un espace et un temps particuliers n'exclut pas les contacts et les insertions extérieurs, mais elle est dominante parce qu'elle tend à subordonner et à imposer ses prérogatives aux autres lieux et temps sociaux. Adopter une observation synchronique des enquêtés qui englobe leurs pratiques «hors champ », scolaires, familiales et amicales, constitue un outil d'investigation puissant pour comprendre l'expérience de formation. En effet, c'est aussi sur ces différentes scènes sociales que prend sens leur ascension sportive, d'où l'intérêt de reconstruire aussi ces engagements, en reprenant les mots d'Olivier Schwartz, «comme un «fait social total» pour pouvoir les comprendre comme faits subjectifs» avec le souci de les rapporter «aux univers sociosymboliques pertinents, c'est-à-dire à ceux dans lesquels ils sont effectivement vécus » (Schwartz, 1995, 296-297).

\subsubsection{L'école à l'épreuve de l'engagement footballistique}

Etant donnée la précocité de la formation, celle-ci engage les apprentis footballeurs dans un double cursus, sportif et scolaire. Parallèlement à l'apprentissage sportif, ils poursuivent leurs études au sein d'établissements scolaires qui offrent la possibilité d'aménagements de l'emploi du temps (un collège, un lycée professionnel, un lycée général et 
technologique) et permettent ainsi la réalisation d'un double cursus. L'analyse des parcours scolaires effectuée sur la base des entretiens et l'analyse des dossiers scolaires aboutit à deux constats principaux.

Tout d'abord, l'étude des parcours avant l'entrée dans l'apprentissage sportif montre que, contrairement à une idée répandue selon laquelle le désintérêt scolaire serait le moteur principal des investissements footballistiques, fragilité des perspectives scolaires et investissement footballistique sont très loin d'aller systématiquement de pair. Seulement un enquêté sur cinq a connu des difficultés scolaires nettes au moment de l'engagement. Les résultats à l'école primaire, en particulier, montrent que la trajectoire scolaire de ces apprentis footballeurs est assez rarement marquée par des difficultés précoces. En fin de cycle primaire, seulement trois d'entre eux sur une population de quarante-trois cas accusaient un retard (d'une année). Huit joueurs sur dix n'ont aucun retard scolaire au moment de l'entrée en préformation (au club ou dans un centre fédéral) ou en formation alors que le recrutement social de cette population reste plus populaire que celui des sportifs de haut niveau (Fleuriel, 2004, 76). Les enfants des milieux populaires sont, en effet, légèrement sur-représentés (57,5\% de père ouvrier ou employé) (2) même si les fils des cadres et professions intellectuelles supérieures sont loin d'être exclus $(19,1 \%)$. C'est alors au regard de ces parcours préalables qu'il est possible d'analyser leur investissement scolaire une fois franchie la porte d'une formation professionnalisante.

Il est possible de distinguer trois temps dans ces parcours qui, malgré les variations liées aux dispositions scolaires préalables inégales, témoignent d'une tendance au désinvestissement scolaire. La grande majorité des joueurs a suivi une partie de sa scolarité du premier cycle des études secondaires au sein du système aménagé qui débute avec la classe de cinquième. En effet, $80 \%$ des enquêtés sont passés par la préformation et l'âge moyen d'entrée au club se situe entre 13 et 14 ans. Les années de collège et de préformation se caractérisent par une intégration des logiques sportive et scolaire, le travail scolaire est vécu comme une charge «normale» par les jeunes du club. Leurs résultats (notes, gratifications et sanctions) sont dans une assez grande continuité avec les années antérieures dans un cadre où les attentes parentales comme celles du club et de son organisation (surveillance importante des résultats scolaires, internat au collège, temps sportif minoritaire) soutiennent l'investissement scolaire. Rares sont les cas de décrochages rapides après l'entrée en formation. Les effets croissants de l'emprise sportive ne sont que progressifs et n'empêchent pas la majorité des enquêtés de s'orienter vers une préparation au baccalauréat. Mais dès l'issue de la seconde de détermination, l'intériorisation des contraintes sportives pèse sur 
l'orientation. Ainsi, même s'il n'existe dans le dispositif qu'un seul type de baccalauréat technologique, celui-ci accueille, de loin, le plus grand nombre d'apprentis : plus de la moitié des membres du lycée général et technologique (56\%) s’y sont orientés (3). La récurrence de cette orientation chez les apprentis est un premier indicateur des effets de l'engagement sportif sur les ambitions scolaires des joueurs qui trouvent dans cette filière une opportunité de poursuivre leur cursus vers le baccalauréat tout en limitant l'investissement nécessaire. Ils peuvent alors, comme cet enquêté, justifier cette orientation par la recherche d'une voie moins exigeante :

«En fait on est beaucoup en STT parce que c'est là que ça demande le moins de travail, donc on a plus de facilité. Alors que y a Laurent, ouais j'crois qu'il y a que lui ici au Centre qui est en S, donc tous les autres en STT, et lui vraiment tous les soirs il galère, c'est vraiment, on le voit jamais quoi, il est à fond dedans, j'sais pas comment il fait d'ailleurs. Et bon souvent quand tu sors de l'entraînement t'es vraiment fatigué, les devoirs... Tu les fais mais bon une heure après c'est bon... Vu qu'en STT, on nous donne pas trop de devoirs, c'est pas trop chargé, c'est pour ça qu'on est tous en STT » [Elève de première STT, 17 ans, fils d'un ouvrier qualifié et d'une mère sans profession].

De manière générale, les années de formation, et donc souvent de lycée, se distinguent par une concurrence accrue entre les deux logiques de formation. Même si l'attrait du baccalauréat demeure, notamment auprès de ceux aux meilleurs parcours scolaires, les résultats sont déclinants. Au final, 56,4\% des apprentis ont obtenu le BEP ou un baccalauréat au terme de leur cursus (environ un tiers ont acquis le baccalauréat). Dans un contexte d'intensification des contraintes sportives, l'école tend à devenir une contrainte concurrente de la «passion » sportive. Ce mouvement s'intensifie à la fin du cursus de formation et l'arrêt de la formation scolaire après la classe de terminale, c'est-à-dire pour la dernière année d'apprentissage au Centre, devient une norme largement répandue parmi les joueurs du club. Cette année est perçue comme un temps à consacrer au football, excluant l'engagement scolaire, et le baccalauréat constitue très souvent, pour les élèves parvenus jusqu'à ce seuil, la fin de la conciliation possible entre les deux activités. Le football impose progressivement son propre calendrier et rend ainsi l'absence d'études supérieures acceptable. Pour Frédéric par exemple, les études supérieures constituaient la voie «normale » à l'intérieur de sa famille (ses parents sont médecins, sa sœur est en école de commerce), mais son engagement footballistique a, selon ses dires, modifié ses perspectives : «Sans le foot, j’aurais ... ben j'aurais fait des études comme tout le monde quoi, j'aurais continué. Mais là avec le foot... ouais, j'suis pas motivé par les écoles en fait. J'préfère me concentrer à fond sur le foot». 
L'arrêt de la scolarité après le baccalauréat parvient ainsi à s'imposer de manière très massive jusque dans les familles les plus dotées en capitaux scolaires et cette tendance illustre bien la double dimension de l'illusio footballistique, entendu «au sens d'investissement dans le jeu et les enjeux, d'intérêt pour le jeu, d'adhésion aux présupposés - doxa - du jeu» (Bourdieu, 1979, 111). S'il constitue une véritable source d'énergie et d'engagement dans les enjeux footballistiques, il est également le moteur d'une indifférence accrue à d'autres formes d'intérêts, en particulier scolaires. L'expression, récurrente en fin de formation, d'une lassitude à l'égard de l'école («j'ai de moins en moins envie» disent-ils souvent), le sentiment d'avoir de moins en moins «la tête à ça», le souhait de ne plus « se prendre la tête avec ça » sont les témoins de leur absorption progressive par les enjeux sportifs. De la même manière, la difficulté pour la grande majorité d'entre eux à se projeter dans un univers professionnel non-sportif illustre cette intériorisation de la vocation (Sorignet, 2004).

\subsubsection{L'engagement sportif, la famille et les « amis »}

La force de l'engagement sportif modifie également la place des apprentis au sein de leurs réseaux de relations familiales et amicales. L'observation des relations familiales et amicales des jeunes footballeurs montre, tout d'abord, de quelle manière elles participent au renforcement de l'identité sportive. En effet, au sein de la famille comme dans le groupe amical, les apprentis trouvent la reconnaissance de leur statut et de leur « place à part ». En apportant leur contribution propre à cette construction symbolique, ces relations prolongent l'inculcation primaire de la vocation dont ces groupes avaient été le siège durant le parcours amateur. La mobilisation et l'attention familiale au projet professionnel, qui tendent à faire de celui-ci une «aventure familiale » collectivement appropriée, assurent la singularisation du jeune apprenti au sein de la configuration familiale. Assiduité parentale aux rencontres sportives, convergence des conversations familiales autour des aléas footballistiques, renforcement d'une relation masculine privilégiée entre un fils «élu » et un père amateur de football sont des indicateurs récurrents de cette attention qui se noue autour de la trajectoire sportive. La force des attentes que ne manque de produire une telle attention explique la crainte fréquente chez ces jeunes de «décevoir » et cela d'autant plus que ces attentes sont souvent renforcées par une renommée acquise, grâce au football, dans un espace local d'interconnaissance. Les relations de sociabilité des apprentis footballeurs participent également à ce renforcement de l'investissement sportif. D'une part, en entrant dans la formation, les joueurs intègrent un groupe de pairs qui devient le lieu central de leur sociabilité, facilité en cela par la consolidation de l'entre-soi sportif qui s'opère grâce au partage d'un même cadre de scolarité (Bertrand, in press). D'autre part, le maintien de 
relations amicales extérieures qui viennent limiter cette clôture relationnelle autour du football et au sein desquelles leur parcours sportif fonctionne comme un capital symbolique, joue également un rôle dans le renforcement de l'identité sportive. Les parties informelles de football qu'ils entament parfois avec ces «copains »sont, par exemple, des lieux privilégiés d'actualisation d'une place distinctive qui confronte les joueurs à la force de l'intérêt qu'ils suscitent.

De plus, l'analyse de ces relations montre également que les cercles amicaux et familiaux sont amenés à se convertir, de manière différente, en espaces de repli. Précarité de leur position sportive et concurrence interne tendent à faire de la famille un lieu de refuge affectif et symbolique. De la même manière, la sociabilité amicale est souvent vécue comme une échappatoire, elle fonctionne alors comme un «troisième lieu» (Renahy, 2005), un espace intermédiaire entre l'institution footballistique et les relations familiales qui autorise la sortie intermittente de l'emprise sportive parce qu'elle permet de «parler d'autre chose » ou de «changer d'air ». Enfin, le poids des contraintes sportives transforme les retours au foyer familial et les incitations amicales en occasions de relâchement et d'actualisation de goûts et dispositions inhibés par le cadre professionnel. Plaisirs d'une cuisine familiale non-conformes aux exigences sportives ou sorties entre «jeunes» sont des exemples parlants de ces suspensions provisoires de l'ascétisme sportif à l'intérieur d'un engagement générant souvent tensions et frustrations. Lieux de renforcement de l'identité sportive, de repli et de compensation, les relations familiales et amicales illustrent bien, comme le montre également l'évolution des rapports à la scolarité, de quelle manière le poids l'engagement sportif implique une recomposition des appartenances extérieures. C'est donc aussi au croisement de ces appartenances que se construit l'expérience de la formation.

\section{La socialisation à un autre regard sur la pratique : la carrière}

\subsection{Le double temps sportif : entre combat collectif et carrière individuelle}

L'apprentissage du football professionnel exploite et renforce une adhésion à la cause footballistique capable de soutenir une mobilisation intensive et durable. Ce travail d'inculcation passe par la production d'un attachement à des enjeux spécifiques. Or il convient de souligner que l'entrée et la vie dans cette «bulle»sont, en réalité, structurées par l'intériorisation d'un double calendrier. Les apprentis sont ainsi portés à s'engager dans une 
double série d'enjeux par lesquels se construit leur adhésion à cet univers et se transforme leur rapport à la pratique.

\subsubsection{Le renforcement des dispositions compétitives}

La formation est, tout d'abord, le lieu où ils sont familiarisés à une immersion continue dans la compétition et où, en d'autres termes, se renforce leur disposition compétitive. Deux dimensions sont révélatrices de cette appropriation du jeu. D’une part, ce renforcement passe par la capacité de l'apprentissage à placer les joueurs dans l'attente et la préparation permanente de la compétition. En faisant du match l'aboutissement et le temps fort autour duquel s'organise la semaine et de la saison sportive la structure principale de l'année, la formation enjoint à la mobilisation compétitive. Mais l'inculcation d'une manière de s'approprier le jeu passe également par la valorisation d'un rapport au match marqué par ce que l'on peut nommer, en reprenant les termes indigènes, une «culture de la gagne ». La rencontre sportive est construite et appropriée comme une épreuve agonistique pour laquelle l'agressivité devient une vertu centrale et complémentaire de la pugnacité. Comme le révèle les observations, «être méchant », «bagarreur », «teigneux », «accrocheur », ne pas être «trop gentil », avoir de la «hargne », sont des qualités exigées avec récurrence par les entraîneurs durant les entraînements alors que l'opposition est régulièrement mise en scène comme une mise à l'épreuve de l'honneur et de la virilité. L'apprentissage sollicite ainsi une disposition orgueilleuse en permettant la sublimation de la conquête d'un capital sportif en une défense virile de l'honneur.

De plus, cet apprentissage de la mobilisation compétitive est, indissociablement, une socialisation au combat collectif. Le match constitue le terrain privilégié d'une solidarité agonistique et de l'actualisation d'un «esprit d'équipe ». Abnégation, discipline collective, insertion dans une division du travail footballistique sont exigées et renforcées par les éléments de ritualisation du collectif qui entourent le moment du match. Repli sur un entre-soi compétitif avant la rencontre, effacement des signes corporels individuels, organisation collective des mouvements (échauffements, sorties des vestiaires, etc.), expression collective des émotions, sont autant de normes de comportement qui incitent les joueurs à faire équipe «par corps ». De la même manière, l'apprentissage de la coordination dans le jeu, la constitution de savoir-faire coopératifs s'appuient sur l'injonction à une morale collective de la solidarité que vient renforcer un ensemble d'habitudes qui font de la compétition un temps collectif et du match l'espace sanctuarisé de l' « esprit d'équipe ».

\subsubsection{L'appropriation d'une concurrence individualisante}


Cependant, l'organisation et le mode de fonctionnement de l'institution, même s'ils donnent une place à la lutte pour les titres parce qu'elle prépare ses membres au combat sportif, ont aussi pour vocation la sélection progressive des apprentis. Ainsi, à l'incertitude du débouché final, la signature d'un contrat professionnel, s'ajoute l'écrémage progressif de la population d'apprentis. Pour cette raison, la sélection et la mise en concurrence sont à l'origine d'enjeux internes qui structurent un second calendrier d'échéances individuelles auxquelles s'ajustent les jeunes joueurs. Au gain d'épreuves sportives s'ajoute l'inquiétude du devenir dans le club alors que celui-ci organise un renouvellement important de ses effectifs. Forte lors de la période de préformation en raison d'un recrutement constant (lors des deux premières années, par exemple, un quart à un tiers des équipes est renouvelé en fin de saison), la sélection se poursuit lors de la formation par la réduction progressive de la population (d'une vingtaine de joueurs à quinze ans, l'effectif passe à une dizaine en fin de cursus). La fin de saison n'est donc pas seulement vécue dans l'attente des phases finales des compétitions mais également dans celle des évaluations individuelles qui décident de l'avenir dans le club. De la même manière, les joueurs vivent les semaines dans l'attente, plus ou moins anxieuse, de l'annonce de la composition de l'équipe et dans l'espoir d'une titularisation. En raison des effectifs de chaque génération, la concurrence est, en effet, une condition quasi-quotidienne des apprentis. Pour chaque match ne sont titulaires qu'environ la moitié des joueurs du groupe et sont exclus de toute participation environ un tiers de l'effectif. Les joueurs se trouvent engagés dans une course au «temps de jeu » dans laquelle ils intériorisent l'impératif concurrentiel selon lequel «il faut gagner sa place » comme ils le répètent souvent. La comptabilisation précise qu'ils font des titularisations, comme des signes d'élection particuliers (surclassement, capitanat), illustre bien leur immersion dans cette lutte individuelle. Leurs espoirs pour les saisons à venir sont alors doubles, compétitifs (remporter un titre national par exemple) et internes (obtenir des titularisations, être conservé par le club). Soumis à des évaluations individualisées et immergés dans cette concurrence, les apprentis intériorisent une nouvelle propension à percevoir la pratique en termes de carrière individuelle. Le désir d' «avoir du temps de jeu », régulièrement annoncé par les joueurs comme un de leurs objectifs, est révélateur de cette intériorisation. Parler en ces termes des matchs de compétition, c'est utiliser une catégorie de perception porteuse d'une représentation individuelle de la formation. Ce ne sont donc plus seulement la victoire et le résultat qui importent, mais aussi la participation à la rencontre, la performance individuelle et, au final, l'évaluation de l'entraîneur. Les apprentis intériorisent donc une perception de la pratique qui, ajustée aux lois de cet espace, est vécue comme une rupture avec les modalités anciennes de 
jeu. Ici on travaille «pour soi », voire «chacun pour soi» disent-ils souvent quand ils décrivent les différences principales avec leurs clubs amateurs précédents. Les joueurs sont ainsi familiarisés à une compétition devenue interne qui tend à produire un rapport concurrentiel aux pairs et constitue, parallèlement, un ressort puissant du pouvoir des formateurs.

\subsection{Une initiation précoce au mode de régulation du marché du travail sportif}

La confrontation à cette lutte individuelle au sein du club tend à transformer le regard sur la pratique. Cette transformation est renforcée par une initiation précoce au mode de régulation du professionnalisme. Les apprentis sont confrontés à des conditions nouvelles (le salariat) et à des acteurs nouveaux (les agents) qui participent à la construction de leur rapport au métier.

\subsubsection{La généralisation des contrats}

Une fois passée la barrière de la préformation, les apprentis connaissent, pour la plupart d'entre eux, un nouveau statut, celui de salarié du club. Les contrats de formation, définis par la Charte du football professionnel qui fait office de quasi-convention collective, se généralisent à l'intérieur du club. Celui-ci salarie une trentaine de joueurs et, dès l'âge de 17 et 18 ans, ce sont environ huit apprentis sur dix qui sont liés par un contrat «aspirant », «stagiaire » ou «espoir» (4). Ceux-ci leur assurent des émoluments croissants (le salaire minimum défini par la Charte est d'environ 600 euros mensuels pour un «aspirant », 1000 euros pour un «stagiaire» et 1500 pour un «espoir»). Par cette gestion contractuelle de ses effectifs, le club se protège contre les départs en cours de formation ou à l'issue de celle-ci, les joueurs étant contraints de signer leur premier contrat professionnel dans leur club formateur (même si cette contrainte ne s'applique pas aux clubs étrangers). Or du fait de sa généralisation dans le club, la signature d'un contrat de formation devient un jalon central dans le cursus des apprentis. Les contrats deviennent des enjeux structurants pour la carrière des joueurs en créant une hiérarchie entre les apprentis et participent du calendrier des échéances individuelles. Pour tout apprenti, signer un contrat devient un objectif important car il constitue une consécration et un étalon de sa propre valeur à l'intérieur du club. Le contrat est vécu comme le signe d'une « confiance » du club, comme un gage, même fragile, pour un avenir toujours incertain. La rétribution financière n'a donc pas uniquement une valeur en soi mais elle est dotée d'un sens sportif parce qu'elle est porteuse d'un avenir possible. La hiérarchie sportive se concrétise en une hiérarchie économique, car les élections et la reconnaissance de la valeur footballistique prennent aussi une forme monétaire. En faisant du salaire un principe de classement et en utilisant aussi l'argent comme outil de sanction ou de 
récompense (sanctions des manquements au règlement ou en cas d'《avertissement» scolaire », primes pour le gain de compétitions nationales), la formation socialise ainsi les joueurs à un espace et à un mode de relation au club où les gains financiers constituent un mode de régulation.

\subsubsection{L'appropriation d'une nouvelle contrainte du métier : «prendre un agent »}

Tout comme les contrats de formation, l'inflation du recours aux agents sportifs est un indicateur, tout en étant un de ses vecteurs, de l'appropriation par les apprentis footballeurs des règles du marché du travail sportif. Les évolutions de ce marché ont fait des agents sportifs un recours quasi-indispensable pour les joueurs professionnels et leur nombre a connu une croissance importante (5). Le développement de ce métier affecte également la formation, la population des apprentis constituant une cible pour ces agents qui peuvent espérer une rémunération lors de l'éventuelle signature du premier contrat professionnel. Souvent sollicités, la grande majorité des apprentis (soit environ huit sur dix) s'est liée à un agent et ils ont acquis la conviction qu'il s'agissait d'une relation presque incontournable pour mener à bien leur projet. Ils attendent de leur part une connaissance experte du marché (notamment pour la négociation des contrats) et espèrent que leur capital relationnel pourra leur procurer une voie de sortie en cas d'éviction du club. Ils sont ainsi initiés, au prix d'une délégation auprès d'un intermédiaire, aux prémices d'une gestion individuelle de la carrière et apprennent à la penser en rapport à un marché. Cette initiation prend d'autant plus de poids que le joueur a acquis une renommée sportive provoquant des sollicitations qui le mettent en contact avec l'espace concurrentiel de la formation et du marché du travail. On comprend dès lors que, parce qu'ils contribuent à produire «une conscience très forte de la notion de carrière » (Slimani, 2000, 217), les agents soient régulièrement la cible des regrets et critiques des entraîneurs et formateurs. «Certains sont biens » disent-ils souvent, mais ils regrettent la place prise par leur activité et usent parfois d'un vocabulaire sévère à leur encontre («parasite », « requin »). Parce qu'elle constitue une concurrence à leur propre pouvoir et à leur monopole de regard expert sur les joueurs et parce qu'elle contribue à alimenter la crainte d'une autonomisation des joueurs par rapport au club, la présence des agents sportifs est fréquemment décriée. Le relatif détachement à l'égard du club que produit cette initiation aux mécanismes du marché footballistique ne peut manquer de heurter les entraîneurs qui, comme cet ancien «pro » du club, ont connu un autre «état du marché ». Formé au club dans les années 1970, il regrette logiquement le rapport de ses élèves à celui-ci : « On est dans notre maison, on est chez nous. Tu vois l'écusson, c'est pas l'écusson d'un autre, c'est le nôtre quoi. Moi j'ai joué ailleurs, c'était pas pareil. (...). Mais c'est plus important pour les vieux, tu vois, ça c'est une autre différence, ça a 
changé. Nous, on était maillot, notre putain de maillot c'est quelque chose. Alors qu'ils s'en foutent les autres, ils savent qu'ils vont faire dix clubs dans leur carrière peut-être. Donc ils ont plus l'identité du tout. Ils sont formés là, mais j'en vois qui sont ici en formation, ils sont supporters de Marseille !».

Au cours de la formation, les apprentis sont ainsi amenés à s'approprier une tension qui traverse la définition du métier. D'une part, ils apprennent à se mobiliser dans un effort collectif pour faire face aux exigences de la compétition dans laquelle ils sont appelés à exceller. Mais, d'autre part, ils intériorisent une perception de la pratique comme carrière individuelle qui se construit dans la concurrence interne et l'insertion sur un marché individualisant et qui rend difficile la production d'un attachement durable au club. Cette tension constitue alors une dimension centrale de l'expérience de ces «nouveaux» footballeurs, et cela d'autant plus que la valorisation de l'«esprit d'équipe » permet de maintenir, en partie, l'ambivalence d'une pratique dans un univers social qui cultive l'ambiguïté entre le métier et la «passion »(Roderick, 2006). La question de l'appropriation d'une formation individuelle dans un sport collectif n'est donc pas simplement d'ordre technique mais également symbolique.

\section{Conclusion}

La littérature sociologique sur le football s'étant majoritairement orientée vers l'analyse du spectacle et des supporters qu'il génère, les footballeur professionnels sont le plus souvent envisagés comme les support de jeux de symbolisation et d'identifications (locales ou nationales par exemple), au risque de désincarner l'exercice de ce métier. Or l'apprentissage de celui-ci à l'intérieur d'une institution enveloppante constitue un terrain idoine pour étudier de quelles manières s'incorpore le monde social. L'analyse de la sociogenèse d'un illusio footballistique, par lequel cet univers s'attache les services d'individus engagés, est favorisée par un dispositif méthodologique qui, parce qu'il peut s'appuyer les travaux socio-historiques existants, combine une approche à diachronique intégrant les parcours antérieurs et une attention portée à la pluralité des appartenances présentes. Le point de vue synchronique permet, en particulier, de souligner les relations dialectiques entre les sphères d'activités ou de relations (rapports familiaux et amicaux, univers scolaire) qui interagissent dans la production des itinéraires des apprentis footballeurs et de quelles manières ces relations évoluent au cours du cursus.

Au final, se dégage ainsi une socialisation qui se caractérise par sa capacité à produire un engagement qui est à la fois intensif, vocationnel, et fortement affecté par son incertitude. 
Si cet apprentissage exige un investissement important, il fait également de la formation une expérience incertaine, concurrentielle et individualisante. Cette préparation au métier de footballeur constitue souvent l'expérimentation d'une vie «sous pression » qui est d'autant plus capable de produire un rapport tendu et inquiet à l'avenir que son mode de fonctionnement rend les trajectoires incertaines et génère fréquemment en son sein des parcours marqués par des oscillations (déclins ou ascensions) rapides et intenses. La conjugaison d'une forte identification au métier de footballeur et d'une fragilité de cette appartenance constitue alors des conditions idoines pour produire des êtres «en tension », facilitant ainsi le pouvoir d'emprise de l'institution.

\section{Notes}

1. Si la formation est progressivement devenue un passage quasi-obligé pour l'accès au marché du travail footballistique, ses débouchés restent très incertains. Les centres de formation produisent un grand nombre de laissés-pour-compte : selon l'UNFP, le syndicat des joueurs, seulement $20 \%$ des joueurs issus des centres de formation accèdent au professionnalisme. Parmi notre population de joueurs déjà fortement sélectionnés car étudiés en fin de formation, environ $44 \%$ sont sous contrat dans un club professionnel au moment de la saison 2006-07, c'est-à-dire une, deux ou trois années après la fin théorique de la formation $(n=29)$.

2. Les apprentis ayant une mère employée ou ouvrière sont eux-aussi majoritaires $(65,8 \%)$ (père : 47 cas ; mère : 45 cas). Le poids des catégories ouvrière et employé dans la population active est de $51,1 \%$ pour les hommes et de $60,4 \%$ pour les femmes [Population active (15-59 ans) : Enquête emploi 2003 (INSEE)] et celles des cadres et professions intellectuelles supérieures de 16, $2 \%$ (hommes).

3. Par comparaison, rappelons ici qu'à l'issue de la seconde de détermination, les statistiques nationales révèlent que l'orientation dans la filière technologique est minoritaire (environ $28 \%$ des élèves entrant en première) et que la voie générale est largement privilégiée (environ 72\%) [MEN-DEP, Repères et références statistiques, Édition 2006].

4. Au sein des trente-deux centres de formation agréés aujourd'hui en France, on compte environ mille sept cents joueurs de quinze à vingt ans liés à leur club par une convention de formation (1732 pour la saison 2005-06) et dont un peu plus de la moitié sont sous contrat avec le club (53\% en 2005-06).

5. La Fédération internationale dénombrait 39 agents (individus ou sociétés) en France en 1999 et 142 en 2005. 


\section{Bibliographie}

- Bertrand, J. (2008). La fabrique des footballeurs : analyse sociologique de la construction de la vocation, des dispositions et des savoir-faire dans une formation au sport professionnel. Thèse de doctorat de sociologie et anthropologie non publiée, Université Lyon II, Lyon.

- Bertrand, J. (in press). «Sociabilité et vocation sportive : des apprentis footballeurs parmi leurs pairs lycéens ». Actes du $3^{\text {ème }}$ Congrès de la Société de sociologie du sport de langue française, Nantes, Octobre 2005.

- Bourdieu, P. (1979). Le sens pratique. Paris, Minuit.

- Brissonneau, C., Aubel, O., Ohl, F. (2008). L'épreuve du dopage : sociologie du cyclisme professionnel. Paris, PUF.

- Faure, J-M., Suaud, C. (1999). Le football professionnel à la française. Paris, PUF.

- Fleuriel, S. (2004). Le sport de haut niveau en France : sociologie d'une catégorie de pensée, Grenoble, PUG.

- Lahire, B. (2002). Portraits sociologiques : dispositions et variations individuelles. Paris, Nathan.

- Lepoutre, D. (1997). Cour de banlieue : codes, rites et langages. Paris, Odile Jacob.

- Papin, B. (2007). Conversion et reconversion des élites sportives. Paris, L'Harmattan.

- Poli, R. (2007). «Migrations de footballeurs et mondialisation : du système-monde aux réseaux sociaux ».M@ppemonde, 88, 12 p.

- Renahy, N. (2005). Les gars du coin : enquête sur une jeunesse rurale. Paris, La Découverte.

- Roderick, M. (2006). The work of professional football. A labour of love ? Londres, Routledge.

- Schwartz, O. (1995). «L'empirisme irréductible». postface de Anderson, N. Le Hobo : sociologie du sans-abris. Paris, Nathan, 265-308.

- Slimani, H. (2000). La professionnalisation du football français : un modèle de dénégation. Thèse de doctorat de sociologie non publiée, Université de Nantes, Nantes.

- Sorignet, P-E. (2004). « Sortir d'un métier de vocation : le cas des danseurs contemporains ». Sociétés contemporaines, 56, 111-132.

- Wahl, A., Lanfranchi, P. (1995). Les footballeurs professionnels des années trente à nos jours. Paris, Hachette. 